\title{
Photocentre offset in ultraprecise astrometry: Implications for barycentre determination and asteroid modelling
}

\author{
M. Kaasalainen ${ }^{1,2}$ and P. Tanga ${ }^{3}$ \\ ${ }^{1}$ Rolf Nevanlinna Institute, University of Helsinki, PO Box 4, 00014 Helsinki, Finland \\ 2 Observatory, University of Helsinki, PO Box 14, 00014 Helsinki, Finland \\ 3 Observatoire de la Côte d'Azur, Laboratoire Cassini UMR 6529/CNRS, BP 4229, 06304 Nice Cedex 04, France
}

Received 2 July 2003 / Accepted 1 December 2003

\begin{abstract}
We investigate the magnitude and characteristics of the photocentre offset phenomenon of small solar system bodies, and compare different scenarios for correcting for it. We discuss the corresponding implications and limitations to the inverse problem of model construction based on astrometry with instruments such as GAIA.
\end{abstract}

Key words. solar system: minor planets, asteroids - astrometry - celestial mechanics - methods: numerical, observational instrumentation: GAIA

\section{Introduction}

The rapid development of astrometric instruments has brought about a new aspect to the astrometry of solar system bodies: in very precise positional measurements, the photocentre and the centre of mass of the target do not coincide. In the next decade, we can expect many satellite- and groundbased observations to reach a positional accuracy better than a milliarcsecond (mas). The defining characteristic of such ultraprecise astrometry is that the actual pixel size can be orders of magnitude higher than the astrometric resolution. There usually are no accurate pixel-resolved data that could support the positional information. Our means of correcting for the photocentre effect are thus very limited particularly for previously unobserved asteroids.

The typical magnitude of the basic photocentre displacement effect for a main-belt (MBA) or near-Earth (NEA) asteroid is of the order of a few tenths of the size of the asteroid, which can translate into tens of milliarcseconds (due to their proximity and high solar phase angles, NEAs may reach larger offsets than MBAs). With an expected practical astrometric precision such as GAIA's roughly 1 mas for asteroids (Hestroffer, personal communication), this is clearly significant.

Constructing a large number of physical asteroid models is an important task of planetary physics. Since several data sources are available, it is also important to know which of these are the most practicable ones. Seldom obtainable space probe observations are the only direct imaging data in the sense that they constitute rather a cartographic than an inverse problem. We know now that, of the other data sources,

Send offprint requests to: M. Kaasalainen,

e-mail: Mikko.Kaasalainen@rni.helsinki.fi
delay-Doppler measurements (Ostro et al. 2002) and photometric observations (Kaasalainen et al. 2002a) are sufficient for building complete asteroid models. The widest range of targets is offered for the latter; however, the latter also need complementary data to improve the scale of resolution. Such data are, e.g., interferometry (particularly that of Hubble Space Telescope's FGS mode; see Hestroffer et al. 2002; Tanga et al. 2001, 2003), stellar occultation timings, and Doppleronly radar observations. Snapshots with space telescopes and adaptive optics are also a practical source of information. Ultraprecise astrometry could be, via the photocentre offset effect, a candidate for this category, so we need to look at it more closely also from this point of view.

The purpose of this short study is to investigate the general implications of ultraprecise astrometry to barycentre determination and target modelling. More specifically, we want to present non-technical and device-independent (and thus necessarily rough) answers to the following basic questions:

1. What is the barycentre accuracy when a) no shape/spin model is used to correct for the photocentre offset, b) a crude model is used, c) a more detailed prior model can be used?

2. How big is the role of the scattering law?

3. How useful is the photocentre offset phenomenon in the inverse problem of constructing physical asteroid models?

We do not concentrate much on the details or restrictions of any particular instrument, though we chose the GAIA mission as a realistic framework for a practical example of instrument resolution. The possible combinations of model/observation parameters are literally endless, and individual cases may vary considerably. Any detailed analysis must thus be case-dependent, 
while our motivation was to find out if there are any generally valid conclusions to be drawn on the hitherto largely unexplored subject (for related studies, see Batrakov et al. 1999; Hestroffer 1998; Lindegren 1977, and references therein).

We present the necessary definitions in Sect. 2, and study the role of the light-scattering model in Sect. 3. Section 4 is concerned with realistic target shape models, while Sect. 5 contains some discussion of the GAIA framework. The implications to the inverse problem are discussed in Sect. 6, and Sect. 7 sums up.

\section{Definitions and photocentre coordinates}

Consider a plane-of-sky coordinate system whose origin coincides with the apparent centre of mass $\left(\alpha_{\mathrm{cm}}, \delta_{\mathrm{cm}}\right)$ of a given object. For sky positions close to the origin, the coordinates $(\Delta \tilde{\alpha}, \Delta \delta)$ of this CM-plane are defined by

$$
\begin{aligned}
\Delta \tilde{\alpha} & =\left(\alpha-\alpha_{\mathrm{cm}}\right) \cos \delta_{\mathrm{cm}}, \\
\Delta \delta & =\delta-\delta_{\mathrm{cm}},
\end{aligned}
$$

where $(\alpha, \delta)$ are the observed spherical coordinates. Also, let the coordinates of the target asteroid's pole direction be given by $\left(\alpha_{\mathrm{p}}, \delta_{\mathrm{p}}\right)$, and those of the asterocentric vector towards the detector by $\left(\alpha_{\mathrm{d}}, \delta_{\mathrm{d}}\right)$. The rotation period of the asteroid is $P$.

Here we take the "photocentre" to be a well-defined, directly determined plane-of-sky point. Mathematically it is a function of the brightness distribution, and its detailed definition depends on the instrument. We have chosen the expected GAIA point-spread-function (PSF) as an example, but in all cases the photocentre is very close to the brightness centre, i.e.,

$x_{\mathrm{pc}}=\frac{1}{L} \int_{A} \boldsymbol{x} \mathrm{d} L$,

where the integration region $A$ is over the illuminated and visible parts of the surface, and a surface patch at $\boldsymbol{x}$ contributes the amount $\mathrm{d} L$ to the observed total brightness (this amount depends on the shape and the light-scattering properties of the surface; see Kaasalainen et al. 2002a). The rotation giving the apparent plane-of-sky coordinates of a point in the asteroid's frame is a constant linear operator, so we have first defined the photocentre $\boldsymbol{x}_{\mathrm{pc}}$ by Eq. (2) as a point in the three-dimensional asteroid frame, and the observed photocentre coordinates $\Delta \tilde{\alpha}_{\mathrm{pc}}$ and $\Delta \delta_{\mathrm{pc}}$ in the CM-plane are then given by, respectively, the $y^{\prime}$ - and $z^{\prime}$-components of

$\boldsymbol{x}^{\prime}=\mathrm{R} \boldsymbol{x}_{\mathrm{pc}}$,

where

$\mathrm{R}=\mathbf{R}_{y}\left(-\delta_{\mathrm{d}}\right) \mathbf{R}_{z}\left(\alpha_{\mathrm{d}}-\alpha_{\mathrm{p}}\right) \mathbf{R}_{y}\left(\delta_{\mathrm{p}}-90^{\circ}\right) \mathbf{R}_{z}\left(-\phi_{0}-\frac{2 \pi}{P}\left(t-t_{0}\right)\right)$,

where $t$ is the time, $\phi_{0}$ and the epoch $t_{0}$ are some initial values, and $\mathrm{R}_{i}(\gamma)$ is the rotation matrix corresponding to the rotation of the coordinate frame through angle $\gamma$ counterclockwise about the positive $i$-axis.

The variations to Eq. (2) due to the instrument are straightforward to include in the analysis, even if they are "smearing" effects caused by the motion of the target during the detector's integration time. The main results of our study are, however, not affected by such variations, so they do not need to be taken into account here.

For any polyhedron, Eq. (2) is given by

$\boldsymbol{x}_{\mathrm{pc}}=\frac{1}{L} \sum_{i} \boldsymbol{x}_{i} \Delta L_{i}$,

where $\boldsymbol{x}_{i}$ is the centroid (geometric barycentre) of facet $i$, and the sum runs through the relevant facets. This form is useful in practical work as shape models are usually rendered as polyhedra (see Sect. 4).

\section{Light-scattering model}

Consider the photocentre offset $d$ for a sphere of radius $R$ observed at a solar phase angle $\alpha$. For geometric scattering law, Eq. (2) immediately gives (see also Lindegren 1977)

$d_{\mathrm{geo}}=R \frac{4}{3 \pi}(1-\cos \alpha)$,

while for a Lambertian sphere this reads

$d_{\text {Lam }}=R \frac{3 \pi}{16} \frac{1+\cos \alpha}{(\pi-\alpha) \cot \alpha+1}$.

The Lambertian offset is quite different from the geometric one, most notably by its linear-like increase. Near zero phase angle, Eq. (7) is approximated by

$d_{\text {Lam }} \approx R \frac{3}{8} \alpha=R 0.0065 \alpha[\mathrm{deg}]$.

Interestingly enough, this approximation is excellent all the way up to solar phase angles as high as $100^{\circ}$. We plot $d_{\text {geo }}$ in dot-dash and $d_{\text {Lam }}$ as a dashed line in Fig. 1, together with the offset $d_{\mathrm{L}-\mathrm{S}}$ from the Lommel-Seeliger law (solid line, Batrakov et al. 1999; Lindegren 1977)

$d_{\mathrm{L}-\mathrm{S}}=R \frac{2}{3 \pi} \frac{\sin \alpha+(\pi-\alpha) \cos \alpha}{\cot \frac{\alpha}{2}-\sin \frac{\alpha}{2} \ln \left(\cot \frac{\alpha}{4}\right)}$

as well as the straight dotted line

$d_{\mathrm{L}-\mathrm{S}} \approx R 0.0062 \alpha[\mathrm{deg}]$,

which is a better general approximation than the $\alpha \rightarrow 0$ limit $\frac{R}{3} \alpha$ of Eq. (9). The Hapke model (e.g., Helfenstein \& Veverka 1989) yields curves between $d_{\mathrm{Lam}}$ and $d_{\mathrm{L}-\mathrm{S}}$; with realistic scattering parameters these curves are usually much closer to the latter. In practice, changing the scattering model typically changes the offset distance less than one percent. It is interesting to note that $d_{\mathrm{L}-\mathrm{S}}$ is completely different from $d_{\text {geo }}$ at low phase angles where Lommel-Seeliger scattering is often considered to be close to geometric scattering.

Since realistic light-scattering models usually give quite similar photocentre locations (cf. Batrakov et al. 1999), we used a linear combination of the Lommel-Seeliger and Lambert laws in the simulations for this paper (with a much smaller weight for Lambert; the general applicability of this scattering model is discussed in Kaasalainen et al. 2001). When analyzing real data, the uncertainty caused by the adopted scattering model is considerably smaller than that caused by the shape approximation or the varying surface characteristics (particularly albedo variegation). 


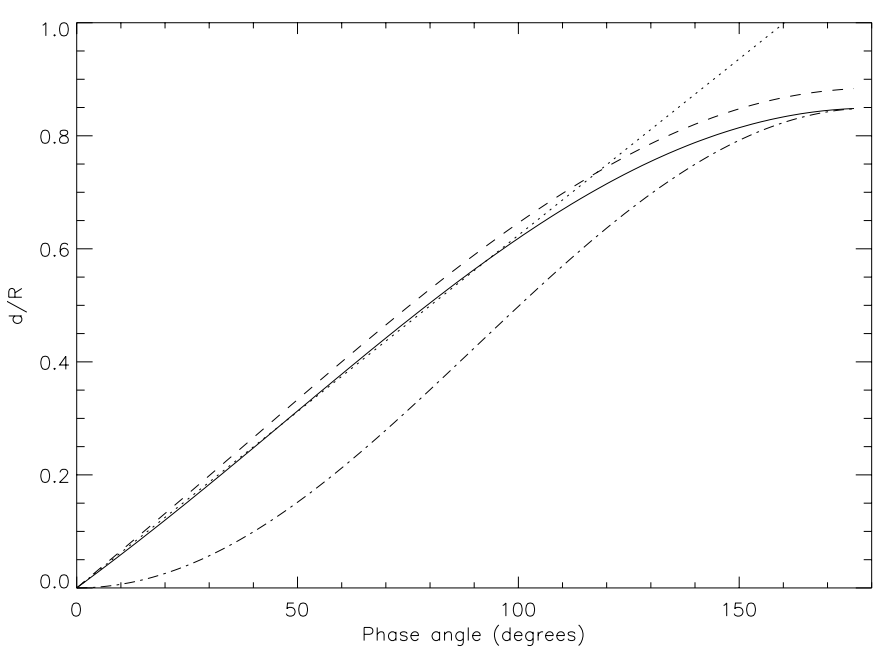

Fig. 1. Photocentre offsets for various light-scattering laws. The solid line corresponds to the Lommel-Seeliger law, the dashed one to Lambertian scattering, and dot-dash to geometric scattering. The dotted line approximates Lommel-Seeliger at phase angles below $100^{\circ}$.

\section{Photocentres of irregular bodies}

We present here some results for three topographic models based on the Galileo images of Gaspra and Ida (Thomas et al. 1994, 1996), and NEAR Shoemaker images of Eros (Zuber et al. 2000); simulations with other realistic models produced very similar results. The model polyhedra consist of thousands of roughly equal-size triangles (for details of tessellation, ray-tracing and brightness computation, see Kaasalainen et al. 2001, 2002a, and references therein).

Figure 2 shows, in a fashion similar to Fig. 1, the average photocentre offset as a function of the solar phase angle. Each average offset was computed over one complete body rotation with the model's rotation axis tilted 30 degrees directly towards the Earth (the model is viewed from 30 degrees above the equator). The Sun's phase angle increases in the plane perpendicular to the one defined by the axis and the direction of the Earth. The solid line corresponds to the Eros model, the dashed line to the lightcurve-based convex Eros model (Kaasalainen et al. 2001), and the dot-dash to a three-axial ellipsoid approximating the shape of the model. The largest radius of the Eros model is normalized to unity. The dotted line portrays the photocentre offset of a sphere approximating the projected area of the model (the projected area of the sphere corresponds to the mean projected area of the target as it rotates in the viewing geometry). Similar phase behaviour is seen at other viewing geometries.

Figure 2, however, tells only a part of the story. An interesting measure is also the dispersion of the photocentre positions from the average location, here shown as standard deviation (computed over a whole rotation of the object) in Fig. 3 with lines corresponding to those in Fig. 2 (the deviation of the sphere model is zero). We can see that at $\alpha \leq 50^{\circ}$ real, "rugged" shapes produce more scattered photocentre locations than unrealistic, smooth ellipsoids. In Fig. 4 we show the phase behaviour and offset scatter for Gaspra and Ida models. At low phase angles the scatter for real shapes is always of the same size as the average offset itself.

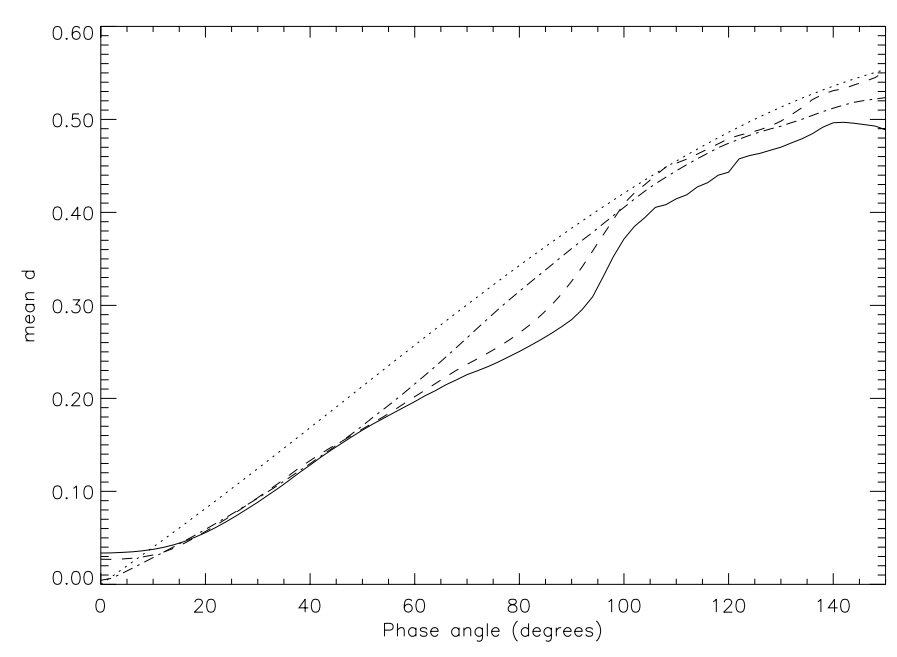

Fig. 2. Average photocentre offsets for an Eros-like simulation model (solid line), its convex representation (dash), an ellipsoid (dot-dash), and a sphere with a radius of 0.68 times the maximal model radius (dot). The offset $d$ is given in units of the maximum radius.

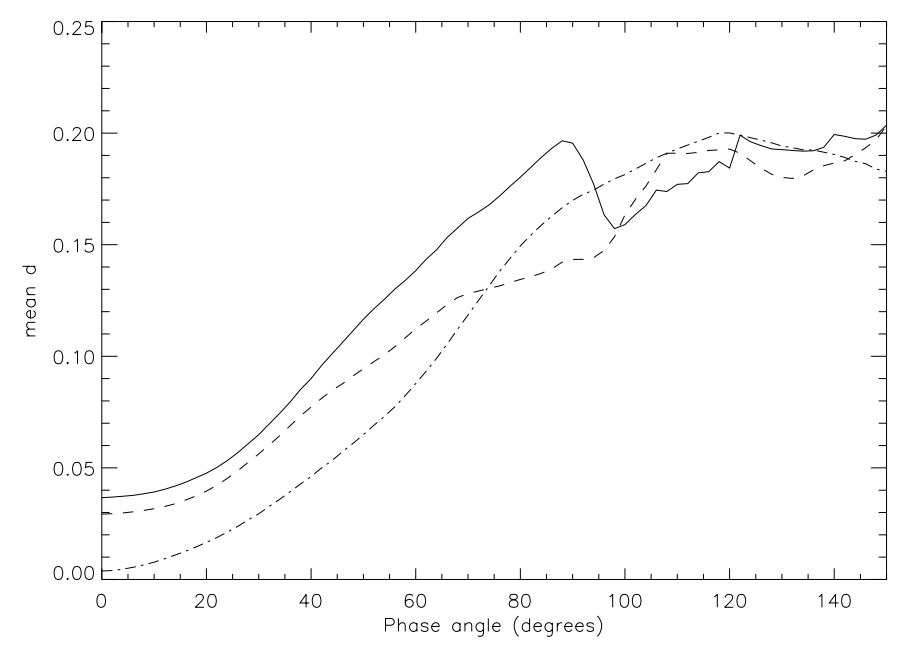

Fig. 3. Standard deviations of the photocentre offsets of Fig. 2.

Even more important than the numerical dispersion value of the photocentre offset is the shape of the photocentre pattern. This looks scattered only for the rugged shapes: for ellipsoids, the photocentre points always tightly concentrate along a plane-of-sky line (the projection of the Earth-asteroid-Sun plane), which is not much more realistic than the point corresponding to a sphere. This is why an ellipsoid is not much better for modelling photocentre locations than a sphere.

In Fig. 5 we show the vertical offset deviations of the three models when the Earth-asteroid-Sun plane line is horizontal (for an ellipsoid the deviation vanishes within the scale resolution). In all cases of varying ruggedness, this deviation is of the same magnitude as that in the horizontal direction. Our simulations thus indicate that the photocentre offset effect has a very strong, pseudorandom component (due to the irregular body surface) in addition to the basic systematic error due to the solar phase angle and the angular diameter of the target.

Note that even large asteroids typically have strong local irregularities even if their global shapes were "pseudoellipsoidal" (Kaasalainen et al. 2002b; Torppa et al. 2003). 


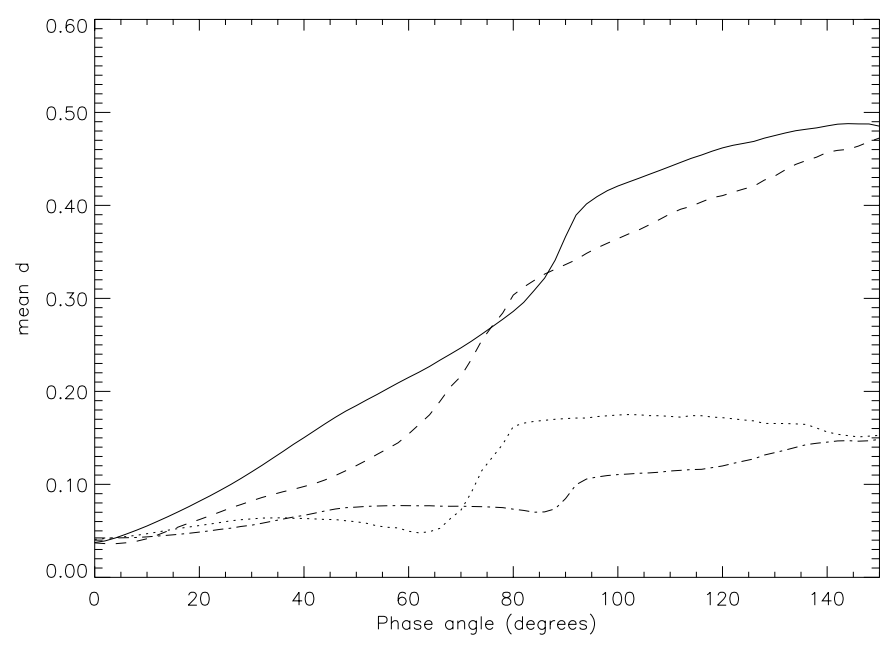

Fig. 4. Average photocentre offsets for Gapra (solid line) and Ida (dash), together with their standard deviations (dot-dash for Gaspra, dot for Ida).

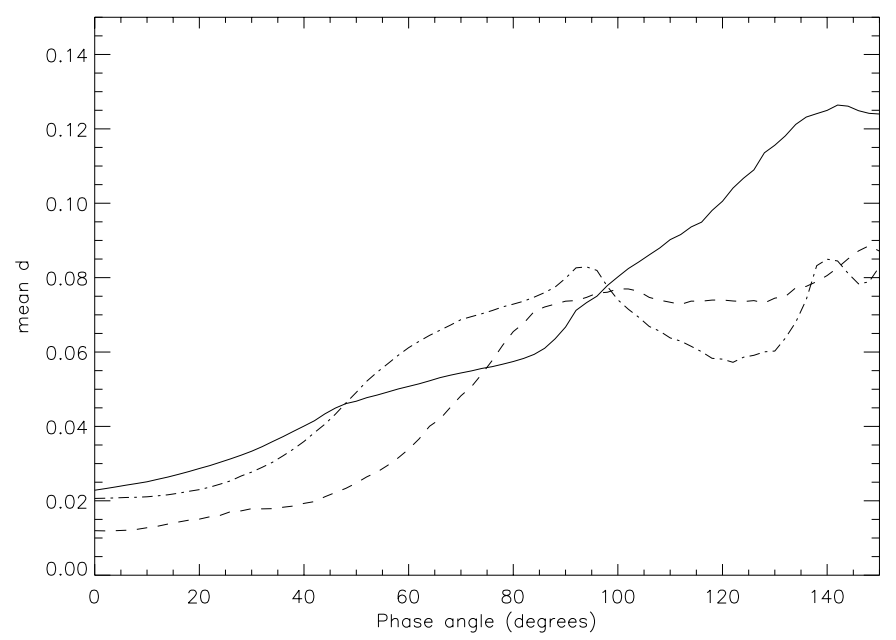

Fig. 5. Standard deviations of the vertical photocentre offsets of Gaspra (solid line), Ida (dash), and Eros (dot-dash).

Simulations with such shapes produce results similar to those above. Also, the irregularity effect is not really dependent on the relative sizes of concavities or other nonconvexities: Gaspra, for example, is only very slightly nonconvex, and completely convex shapes behave in similar fashion.

\subsection{Corrected position}

If there are no physical models of the target at our disposal, it is always possible to use a "zeroth-order" spherical correction obtained from Eq. (10), assuming some $R$ from, e.g., interferometric measurements, rough pixel counts, or observations in the infrared, and taking the photocentre displacement to be in the CM-plane direction of the Sun, i.e.,

$\boldsymbol{x}^{\prime}=d \mathrm{R}_{y}\left(-\delta_{\mathrm{d}}\right) \mathrm{R}_{z}\left(\alpha_{\mathrm{d}}\right) \boldsymbol{E}_{\text {Sun }}$,

where $\boldsymbol{E}_{\text {Sun }}$ is the asterocentric unit vector towards the Sun. Another possibility is to use $d$ as a free parameter, to be iteratively improved simultaneously with the orbit solution. In this

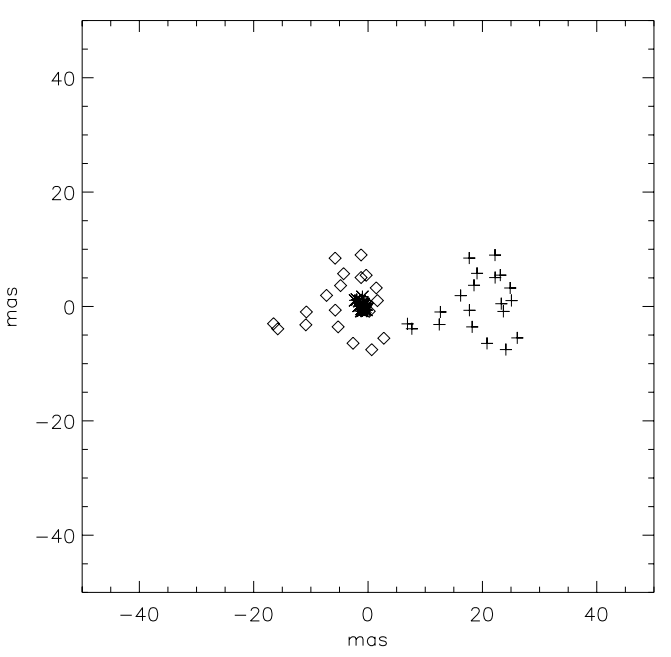

Fig. 6. Homing in on the right centre of mass. Crosses are observed photocentre positions (20 observations during one object rotation), diamonds correspond to a crude correction, and asterisks are the estimated centre-of-mass positions from a previous global shape/spin model. The largest apparent radius of the object (Gaspra model) is 107 mas, and the solar phase angle is $50^{\circ}$.

way it is possible to get a very rough size estimate (cf. Batrakov et al. 1999; Hestroffer 1998).

If there is a previously determined asteroid model available (from, e.g., photometric data, radar, or fly-by images), it should obviously be exploited to minimize the effect of the photocentre offset. For most "modellable" asteroids we have a global shape/spin model that does not include topographic details but is more accurate than an ellipsoidal representation. Now we can determine the centre-of-mass positions as with the crude model since the orbit parameters needed in Eq. (4) are obviously known well enough a priori.

Figure 6 shows the effect of both crude and modelbased corrections. The observed photocentre positions of a Gaspralike model are shown as crosses in the CM-plane of the target. Applying a (somewhat overoptimistically well guessed) crude correction from Eq. (10) shifts these points uniformly to the left (diamonds), i.e., the estimated CM positions are now distributed around the correct centre of mass. This cluster becomes tighter (asterisks) when a better correction from the lightcurve-based global shape/spin model (Kaasalainen et al. 2001) is applied.

Because of the effectively random component of the photocentre offset, a crude correction (by a sphere or an ellipsoid) cannot lower the astrometric photocentre "noise" by more than a half, while a better known prior model can reduce the offset error to some $10 \%$ of the uncorrected level.

\section{Photocentre measurements with GAIA: Practical accuracy}

The GAIA mission is an example of future ultraprecise astrometry. Bodies with angular size of 100 mas will typically have offsets of order 10 mas (for NEAs this can be slightly larger than for MBAs, as the former are expected to be observed at some $20^{\circ}$ higher solar phase angles than the latter that will 
usually be observed at some $10^{\circ}-20^{\circ}$ ). A good prior model can reduce this to about 1 mas, i.e., GAIA's expected practical astrometric accuracy limit for asteroids. Given this limit, the photocentre offset (or the existence of prior models) for small targets less than 10 mas in angular size is not so important for astrometric accuracy any more, particularly as their dimness makes their astrometric resolution poorer than that of the larger ones.

In order to simulate the detection by GAIA of an extended object of complex shape, we used high resolution synthetic images of Eros, and we convolved them with a model of GAIA PSF, obtained by the GIBIS simulator (http://www . ast. cam.ac.uk: 8080/gibis/). The simulated PSF corresponds to a star of colour index $V-I=+1.5$, having no aberration and no noise added. At this stage, we also neglect the effect of smearing due to the apparent motion of the object. The PSF is 4-times oversampled in comparison to the Astrometric Field CCD, supposed to have pixels of a size corresponding to $44 \times 133$ mas, the shortest side being parallel to the scan direction. The only deterioration added over the optically ideal PSF is a smearing of 1 pixel (despite the 4-phase CCD charge transfer) that is introduced to simulate the effect of charge diffusion, scan rate error, etc., as described in the GAIA document SAG-LL-025 (available on request at http://astro.estec.esa.nl/GAIA/) to which the reader should refer for further details. Thus, the accuracy of the photocentre determination is somewhat exaggerated in these simulations.

The asteroid is always considered to have its rotation axis perpendicular to the scan direction, i.e., to be oriented acrossscan. As a consequence, the greatest dispersion in the photocentre measurements is expected to be in the along-scan direction, where the angular resolution is higher.

The simulations were run with different apparent sizes for the object. For this particular example, we chose the solar phase angle $30^{\circ}$ and the aspect angle $30^{\circ}$ as a typically representative observing geometry, but the choice of the geometry does not affect the main result. The shape's major extent was taken to be from 12 mas to 200 mas. The position of the photocentre was estimated by the photometric barycentre of the convolved image. A comparison of the photocentre position with the photocentre of the image at full (virtually infinite) resolution shows, as expected, that for the largest sizes the effect of sampling is negligible, i.e., the sampled image photocentre mimics well the behaviour of the photocentre in the full resolution asteroid model (Fig. 7a). At smaller sizes, when the shortest object extent becomes smaller than the across-scan pixel size, the photocentre displacement collapses along the direction of lower resolution (Fig. 7b) and, in general, the dispersion is always smaller than that of the fully resolved image.

In reality, the strong anisotropy in the dispersion will be broken by the fact that the scan direction changes for each observing epoch. Although some systematic effects could still be present due to the non-uniform probability of all scan directions, we can expect the real GAIA astrometric dispersions of the photocentres to be much more isotropic.

An estimate of the maximum dispersion that can be expected for irregular objects of different sizes can be obtained
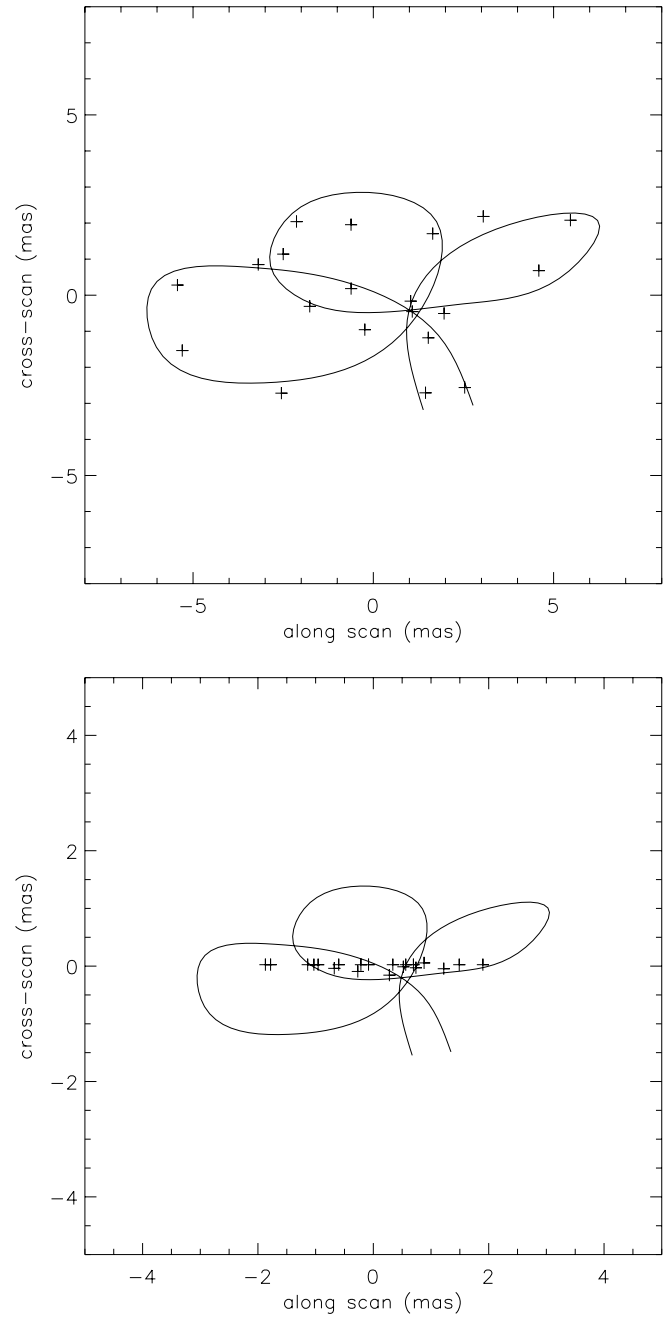

Fig. 7. Photocentre coordinate dispersion in the CM-frame for our simulation model during one rotation at solar phase angle $\alpha=30^{\circ}$. Here the origin has been shifted to the average photocentre position. The solid line corresponds to the theoretical offset (2), while the crosses represent the simulated "readings" by GAIA. The upper graph corresponds to an apparent maximal diameter of 100 mas, while for the lower graph the diameter is 50 mas.

by considering the total range of dispersion. This has been normalized to the object's greatest dimension and plotted in Fig. 8; we can see that the "full resolution" level is achieved at about 100 mas. The figure also clearly shows how the measurement of the dispersion collapses across-scan first due to the larger pixel size in the corresponding direction.

\section{Inverse problem}

Our ultimate goal would be to find the orbit and physical model giving the best fit to $N$ photocentre observations $\left(\alpha_{i}, \delta_{i}\right)$ at epochs $t_{i}$. Our objective function is thus

$\chi_{\mathrm{pc}}^{2}=\sum_{i=1}^{N}\left[\cos ^{2} \delta_{i}\left(\alpha_{\mathrm{pc}}\left(t_{i}\right)-\alpha_{i}\right)^{2}+\left(\delta_{\mathrm{pc}}\left(t_{i}\right)-\delta_{i}\right)^{2}\right]$,

where $\left(\alpha_{\mathrm{pc}}\left(t_{i}\right), \delta_{\mathrm{pc}}\left(t_{i}\right)\right)$ are the modelled positions from Eqs. (5), (3), and the orbital evolution equations for the centre of 


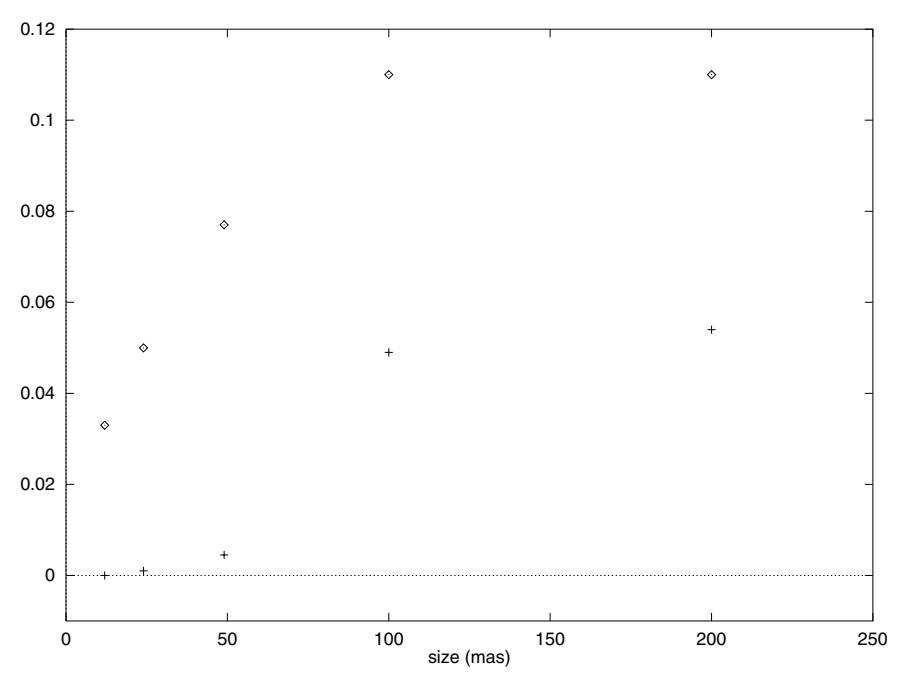

Fig. 8. Photocentre dispersion of simulated GAIA observations as function of the apparent diameter of the object, normalized to object size. Diamonds are for along-scan direction, and crosses for acrossscan.

mass: $\alpha_{\mathrm{pc}}=\alpha_{\mathrm{cm}}+\Delta \tilde{\alpha}_{\mathrm{pc}} / \cos \delta_{\mathrm{cm}}$ and $\delta_{\mathrm{pc}}=\delta_{\mathrm{cm}}+\Delta \delta_{\mathrm{pc}}$. This can be appended with a $\chi_{0}^{2}$ from other data.

The traditional geometric orbital elements are not the best orbit parameters for optimization purposes. It is easier just to use, via Gauss' $f, g$-functions (Danby 1987; a compact presentation is also given in Kaasalainen \& Laakso 2001), the initial Cartesian position and velocity vectors for the first epoch; any alternative elements can always be directly computed from these. This has the added benefit of providing a robust error measure such as the relative error of the initial phasespace point, and the derivatives are also readily available for gradient-based optimization and solution improvement (such as Levenberg-Marquardt; Press et al. 1994). We only use orbit parameters as part of the simultaneous inverse problem: orbit determination based on plane-of-sky barycentre positions is a somewhat separate matter extensively discussed elsewhere (see Bowell et al. 2002, and references therein).

\subsection{Limited information content of photocentre measurements}

Our initial goal was to carry out a sequence of simulations to see whether we could establish a rule-of-thumb scale for the extractable information as a function of the number and other characteristics of observations. Having performed several simulations, we came to the conclusion that (in the general case of a shape with irregularities) such a scale can hardly be established since the photocentre offset effect quite clearly is not a very useful data source as far as the inverse problem of physical modelling is concerned.

There is a twofold mathematical reason to why the photocentre offset cannot provide much modelling information either on its own or even as complementary data. First, the $\chi^{2}$-topography of Eq. (12) is complicated with several local minima. Second, a simple model, such as an ellipsoid, gives a poor fit to the data of a realistic object as described above.
(We use just the minimum seven parameters for an ellipsoidal model: the pole direction $\alpha_{\mathrm{p}}, \delta_{\mathrm{p}}$, period $P$, initial rotation angle $\phi_{0}$ at some epoch $t_{0}$, and the ellipsoid semiaxes $a, b, c$, i.e., including the absolute size scale.) There are numerous different pole and ellipsoid configurations that fit the data just as well as or even better than the actual best ellipsoid and pole.

The inverse problem is thus severely ill-posed, and, worst of all, this is due to fundamental nonuniqueness rather than instability. This happens already when the orbit is assumed completely known, and even with several tens of astrometric observations and various viewing geometries. This is not yet even the realistic case: orbit parameters will necessarily have to be added to the set, and we found that then the inverse problem becomes considerably more nonunique and ill-posed.

These facts mean that photocentre data cannot assist much in physical modelling, either with simple or more complex models. Indeed, we found that ellipsoidal models were not really better than just a simple spherical correction Eq. (11). The orbit parameters are best determined using a given model, either a crude or a more detailed one, for barycentre determination. As far as GAIA is concerned, for example, we can expect to have hundreds of asteroids models available by the time the instrument flies.

We can thus see that although a good physical model of the object is necessary for highly accurate barycentre determination, the converse is hard to attain: photocentre positions do not contain much information on the target itself. Their information content is, particularly at low solar phase angles, considerably lower than, e.g., that of lightcurves (symmetric bodies exhibit no photocentre offset at zero solar phase angle). One could also say that photocentre positions are so sensitive to intermediatescale surface features that global-scale information is largely submerged in the corresponding noise.

Despite the lack of constructive information, several consecutive astrometric observations may be used to rule out a wrong pole solution of an otherwise fairly detailed prior model: we can expect $\chi_{\mathrm{pc}}^{2}$ to be usually higher for the "mirror poles" that may remain in photometric analysis.

\subsection{Binary systems}

For a binary asteroid configuration to reveal itself via astrometric measurements, the system should at least have a certain level of asymmetry: two identical objects revolving round each other give (excluding occultation/eclipse events) exactly the same photocentre offset as a single one. The asymmetry effect is connected with the scaling difference between area and mass. The photocentre offset caused by the relative size scale difference $s$ of the two bodies is proportional to $\left(s^{2}+1\right)^{-1}-\left(s^{3}+1\right)^{-1}$, so it reaches its maximum when $s=1.87$. Thus binary systems with the secondary component about half the size of the primary provide the best chances for the corresponding photocentre offset to be seen.

We found that no simple (seven-parameter) two-sphere binary model could fit the simulated astrometric data of a binary system of two irregular bodies well, i.e., the photocentre "signature" of binary asteroids is certainly not very striking. 
There seems to be a consistent though small fit difference in favour of the binary model as compared to a single ellipsoid model, but this is mostly qualitative as the obtained parameter values are very inaccurate.

The main qualitative difference between the photocentre motion of a single body and that of a binary system (or a strongly asymmetric body) is due to the same reason that causes the "wobble" effect of exoplanetary systems: the photocentre of the target may oscillate around the barycentre instead of staying on one side of it. In the case of asteroids, the problem lies in ascertaining the position of the barycentre, so the wobble does not reveal itself directly. The more there are consecutive observations of a binary system, the better a model that allows the orbit trajectory to travel through the photocentre locations (instead of keeping them on one side of the sky-path) is likely to fit the data.

\section{Conclusions}

We have given here some basic answers to the three basic questions stated at the beginning. It should be emphasized that detailed answers depend on the instrumental implementation and mission parameters: the GAIA and SIM missions, for example, are going to be quite different even though they are both based on precise astrometric techniques.

We have shown that the photocentre offset effect of real(istic) bodies has a pseudorandom component of the same magnitude as the average size of the offset itself. Only a welldetermined model can reduce this "noise" significantly, i.e., to the level of a few percent of the angular diameter (while the level of the uncorrected noise is an order of magnitude higher). This underlines the fact that, for most well observable asteroids, the effective astrometric barycentre resolution with instruments such as GAIA can seldom be better than 1 mas. GAIA will fully resolve the photocentre offset for objects with apparent diameter over 100 mas. The role of the lightscattering model is small, only of order one percent of the offset size. Astrometric observations provide little information for the physical modelling of the target. Asymmetric binary systems may stand apart from single bodies, but their properties cannot be modelled well using astrometric observations alone.

We thus think that future studies of the photocentre effect, aiming at the improvement of astrometric measurements, should build upon the use of prior models for the object shape and spin state. However, particularly for binary asteroids and asteroids approaching simple shapes a photocentre offset correction (better than just a sphere) could be implemented with the aid of simultaneous photometric and coarse-scale pixel information. Such a case is heavily dependent on the details of the observing instrument and mission, and we plan to analyze this in the GAIA framework in a future study.

Acknowledgements. We thank Daniel Hestroffer and Jenni Virtanen for useful comments and discussions, and the referee, Yu. V. Batrakov, for valuable suggestions and comments.

\section{References}

Batrakov, Yu. V., Chernetenko, Yu. A., Gorel, G. K., \& Gudkova, L. A. 1999, A\&A, 352, 703

Bowell, E., Virtanen, J., Muinonen, K., \& Boattini, A. 2002, in Asteroids III, ed. W. Bottke, R. Binzel, P. Paolicchi, \& A. Cellino (Tucson: University of Arizona Press), 27

Danby, J. M. A. 1987, Fundamentals of Celestial Mechanics (Richmond: Willman-Bell)

Helfenstein, P., \& Veverka, J. 1989, in Asteroids II ed. R. P. Binzel, T. Gehrels, M. S. Matthews (Tucson: University of Arizona Press), 557

Hestroffer, D. 1998, A\&A, 336, 776

Hestroffer, D., \& Tanga, P., Cellino, A., et al. 2002, A\&A, 391, 1123

Kaasalainen, M., \& Laakso, T. 2001, A\&A, 368, 706

Kaasalainen, M., Torppa, J., \& Muinonen K. 2001, Icarus, 153, 37

Kaasalainen, M., Mottola, S., \& Fulchignoni, M. 2002a, in Asteroids III, ed. W. Bottke, R. Binzel, P. Paolicchi, \& A. Cellino (Tucson: University of Arizona Press), 139

Kaasalainen, M., Torppa, J., \& Piironen, J. 2002b, Icarus, 159, 369

Lindegren, L. 1977, A\&A, 57, 55

Ostro, S., Hudson, S., Benner, L., et al. 2002, in Asteroids III, ed. W. Bottke, R. Binzel, P. Paolicchi, \& A. Cellino (Tucson: University of Arizona Press), 151

Press, W. H., Flannery, B. P., Teukolsky, S. A., \& Vetterling, W. T. 1994, Numerical Recipes (Cambridge: Cambridge Univ. Press)

Tanga, P., Hestroffer, D., Berthier, J., et al. 2001, Icarus, 153, 451

Tanga, P., Hestroffer, D., Cellino, A., et al. 2003, A\&A, 401, 733

Thomas, P. C., Veverka, J., Simonelli, D., et al. 1994, Icarus, 107, 23

Thomas, P. C., Belton, M. J. S., Carcich, B., et al. 1996, Icarus, 120, 20

Torppa, J., Kaasalainen, M., Michalowski, T., et al. 2003, Icarus, 164, 346

Zuber, M. T., Smith, D. E., Cheng, A. F., et al. 2000, Science, 289, 2097 